\title{
New understanding of phase segregation of bimetallic nanoalloys
}

\author{
Yuen $\mathrm{Wu}^{1,2}$ and Yadong $\mathrm{Li}^{{ }^{*}}$
}

In a recent online publication of Science, Professor Peter Strasser of the Technical University of Berlin, Germany, and his collaborators reported element-specific anisotropic growth of $\mathrm{Pt}$ and $\mathrm{Ni}$ in shaped $\mathrm{Pt}$ alloy synthesis [1]. They showed that the surface steps in the $\mathrm{Pt}_{3} \mathrm{Ni}$ concave hexapod alloy formed in the initial stage of the synthesis were crucial in the segregation of an M-rich $(\mathrm{M}=\mathrm{Ni}, \mathrm{Co}$, etc.) phase in the $\mathrm{Pt}-\mathrm{Ni}$ alloy through step-induced deposition. Controlled synthesis of the segregated phase in the bimetallic system will help in the rational design of new nanostructured materials with desired physical and chemical properties. In addition to new segregated Ni structures on Pt-Ni surfaces, important step-induced layer-by-lay- er growth mechanisms were previously reported by Li's group, and can be tracked back to the work published in the Journal of the American Chemical Society in 2013 [2]. It was reported that the atomic steps on the nanosegregated Pt skin of a Pt-based bimetallic alloy can be used to achieve site-selective nucleation of $\mathrm{Ni}$, resulting in further phase segregation of $\mathrm{Ni}$ in $\mathrm{Pt}-\mathrm{Ni}$ bimetallic nanoalloys. This new intrinsic step-induced growth mechanism enables site-selective nucleation of a third metal $\mathrm{M}$ around the defects, to achieve sophisticated design of more types of phase segregation $\left(\mathrm{Pt}_{3} \mathrm{Ni} @ \mathrm{M}, \mathrm{M}=\mathrm{Au}, \mathrm{Ag}, \mathrm{Cu}\right.$ and $\left.\mathrm{Rh}\right)$.

A series of groundbreaking investigations of the segregation of bimetallic alloys, and closely related catalytic
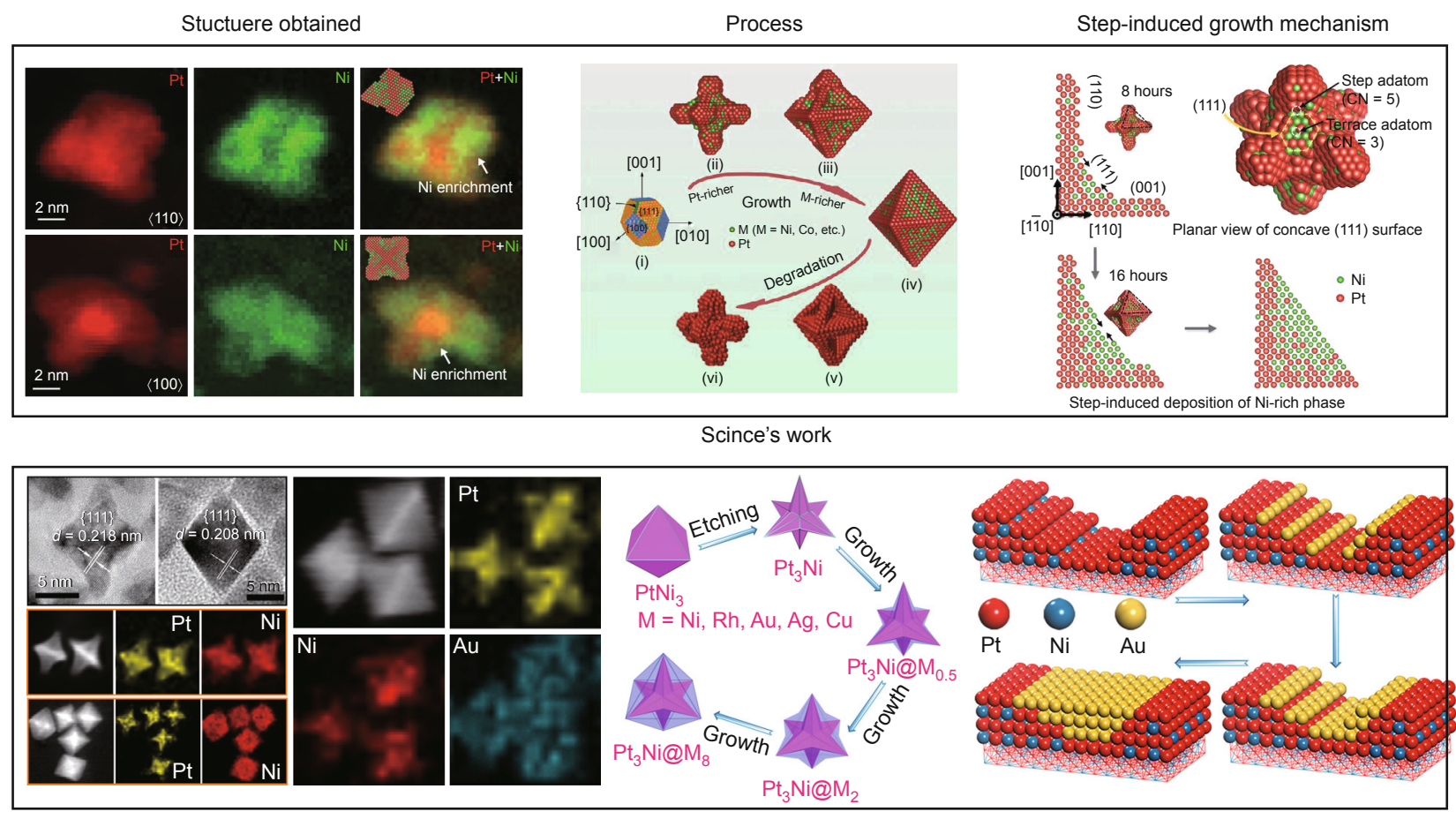

JACS's work

Figure 1 Schemes illustrating the structures, formation, and mechanisms of phase segregation in bimetallic nanoalloy. Adapted with permission from Ref. [1] (Copyright 2014, American Association for the Advancement of Science) and [2] (Copyright 2013, American Chemical Society).

\footnotetext{
${ }^{1}$ United Innovation Center of Catalysis and Department of Chemistry, Tsinghua University, Beijing 100084, China

${ }^{2}$ Center of Advanced Nanocatalysis (CAN-USTC) and Department of Chemistry, University of Science and Technology of China, Hefei 230026, China

*Corresponding author (email: ydli@mail.tsinghua.edu.cn)
} 
studies, are listed here according to the timeline. In 2012, Li's group reported that a concave octahedral Pt-Ni alloy with a Pt-segregated surface can be constructed using a controlled dealloying process [3]. This work has led to the effective design of nanosegregated Pt skins on Pt-based bimetallic nanoalloys using a simple wet-colloid method. Soon afterwards, Strasser and co-workers [4] reported that the inhomogeneous distribution of presynthesized Pt-Ni seeds can be used to produce a Pt-segregated surface on a concave octahedral Pt-Ni alloy. In 2014, Chen et al. [5] reported that a $\mathrm{Pt}_{3} \mathrm{Ni}$ rhombic dodecahedral nanoframe composed of 24 edges and 14 corners can be synthesized under oleylamine-mediated conditions, and enable the maximum use of Pt by a three-dimensional accessible surface. The geometrical and electronic effects induced by the nanosegregated Pt-skin surface substantially improve the activity of this nanoframe catalyst in the oxygen reduction reaction.

In bulk alloys, two metals are usually considered to be homogeneously distributed. However at the nanoscale, the metal distributions differ and the mixed topological structures can be classified as alloys, intermetallics, surface segregated, and partly segregated. Some important factors such as metal bonds, electronegativities, surface energies, and surface-binding agents significantly affect the mixing patterns of bimetallic alloys. It should be noted that the induction of segregation depends on the balance among the factors listed above, as well as on the practical experimental conditions and synthetic strategies. Segregation is common in bimetallic alloys at the nanoscale. The unsaturated binding of surface atoms cannot be compensated for by bulk atoms, so detailed microstructures such as lattice strain and surface energies should be taken into consideration. The distributions of two metals in bimetallic alloy are thermodynamically stable and the surface segregation may occur. In recent years, substantial efforts have been made to tune the phase segregation as well as the composition and atomic ordering of the bimetallic nanoalloys to optimize their chemical activities, especially their catalytic activities.

In situ characterization of the segregated phase of a bimetallic nanoalloy during the catalytic cycle and atomicscale control of segregation in the synthesis of bimetallic nanoalloys are desirable, but challenging. It is difficult to observe and characterize segregation if the resolution of the technique used to distinguish the spatial distributions of two metals is insufficient for observations at atomic level. Recently, the emerging technique of probe-corrected scanning transmission electron microscopy and electron energy-loss spectroscopy has become an effective and powerful method for determining compositional evolution trajectories and segregation with atomic-scale resolution. This new technique shows how two types of metal can assemble and form a well-ordered bimetallic nanostructure. The development of versatile synthetic methods for controlling segregation in bimetallic nanoalloys is also a challenge because of the complex factors that need to be taken into consideration, such as redox potentials, interfacial energies, reduction rates, and capping agents. An understanding of segregation in bimetallic nanoalloys could provide valuable information on tracing the real catalytic centers and help to develop more efficient bimetallic nanoalloy catalysts.

Received 3 January 2015; accepted 4 January 2015;

published online 20 January 2015

1 Gan L, Cui C, Heggen M, et al. Element-specific anisotropic growth of shaped platinum alloy nanocrystals. Science, 2014, 346: $1502-$ 1506

2 Wu Y, Wang D, Chen X, et al. Defect-dominated shape recovery of nanocrystals: a new strategy for trimetallic catalysts. J Am Chem Soc, 2013, 135: 12220-12223

3 Wu Y, Wang D, Niu Z, et al. A strategy for designing a concave Pt$\mathrm{Ni}$ alloy through controllable chemical etching. Angew Chem Int Ed, 2012, 51: 12524-12528

4 Cui C, Gan L, Heggen M, Rudi S, Strasser P. Compositional segregation in shaped Pt alloy nanoparticles and their structural behaviour during electrocatalysis. Nat Mater, 2013, 12: 765-771

5 Chen C, Kang Y, Huo Z, et al. Highly crystalline multimetallic nanoframes with three-dimensional electrocatalytic surfaces. Science, 2014, 343: 1339-1343 